\title{
THE NUMERICAL SIMULATION OF THE SHIELDED HELMHOLTZ COILS SYSTEM MAGNETIC FIELD
}

\author{
Ivan Zatonov*, Pavel Baranov \\ National Research Tomsk Polytechnic University, 634050, Tomsk, Russia
}

\begin{abstract}
This article describes the calculation of the magnetic field in the axial direction for the Helmholtz coil. The inhomogeneity of the magnetic field for this coil system is calculated. Authors defined the functional connection of the magnetic flux density in the system center from the antimagnetic shield thickness in two ways: iron and Finemet alloy shield. The dependency graph of the magnetic field inhomogeneity in the center of shielded Helmholtz coils system from the axial coordinate value is estimated
\end{abstract}

\section{Introduction}

The measurement of the weak magnetic field is crucial for the science and technology application[1-3]. For the weak magnetic field measures the magnetic-test coils, flux-gate meters, non-isotropic magnetoresistive transducers, squids are used.

For the sensor of the weak magnetic field adjustment and calibration measurement it is required to have the magnetic field with high homogeneity. For the calibration devices with the inaccuracy less than $1 \%$ field inhomogeneity should not exceed $0.1-0.3 \%$ in the magnetic field measurement device space.

In the function of homogeneity magnetic field sources solenoids the Helmholtz coils [4] (Figure 1), square coils system, Spheroidal coils [5], multicoil system [6] and others coil systems are used.

The presence of the Earth magnetic field and others magnetic disturbance makes the process of weak magnetic field calibration more difficult and sometimes impossible and it also could lead to inaccuracy appearance. The article presents the analysis of the magnetic field homogeneity with the magnetic flux density equals to $50 \mu \mathrm{T}$ with the magnetic shield from the iron and Finemet alloy material.

\section{The calculation of the magnetic field axial component}

The calculation of the magnetic field inhomogeneity requires the evaluation of the magnetic flux density in the axial direction.

\footnotetext{
*Corresponding author: iaz5@tpu.ru
} 


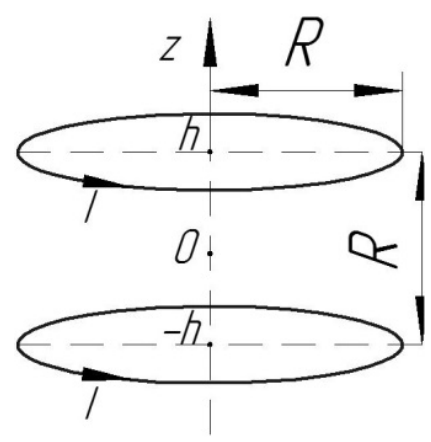

Fig. 1. Helmholtz coils.

According to the Biot-Savart law the magnetic flux density of the Helmholtz coils on the distance ' $z$ ' from the coordinates center can be calculated by the following formula:

$$
B(z)=0.5 \mu_{0} I N R^{2}\left\{\left[(z-h)^{2}+R^{2}\right]^{-\frac{3}{2}}+\left[(z+h)^{2}+R^{2}\right]^{-\frac{3}{2}}\right\}
$$

where $\mu_{0}-$ is vacuum permeapility equals to $4 \pi \times 10^{-7} \mathrm{H} / \mathrm{m} ; I-$ current in the wire; $N$-number of winding; $R$ - coils radius; $h$-distance between coils

For the determination of distance ' $h$ ' in is necessary to find the solution the conditions (2). The second member of the Taylor expansion in the point $z=0$ must be equals to zero:

$$
B^{(2)}(0)=0 \text {. }
$$

The solution of the formula (2) shows that the creation of the inhomogeneity magnetic field requires coils to be at the distance of $\mathrm{R} / 2$ from the center. Taking into account formulas (1) and (2) we have the finite expression for determination of the Helmholtz coils magnetic flux density in the axial direction:

$$
B(z)=0.5 \mu_{0} I N R^{2}\left\{\left[\left(z-\frac{R}{2}\right)^{2}+R^{2}\right]^{-\frac{3}{2}}+\left[\left(z+\frac{R}{2}\right)^{2}+R^{2}\right]^{-\frac{3}{2}}\right\}
$$

Knowing the magnetic flux density spread nature in the direction in the line of ' $z$ ' and the value of flux density in the system center it becomes possible to calculate the field inhomogeneity using the following formula:

$$
\delta_{z}=\frac{|B(0)-B(z)|}{B(0)} * 100 \%
$$

where $B(0)$ - the magnetic flux density in the system center.

This calculation considers the ideal situation when the cross section of the coils tends to zero. In the reality coils have some cross section that influence the spread of the magnetic field. The finite element analysis was carried out in order to take into account coils cross section influence as well as external magnetic field and the magnetic shielding. 


\section{Finite-element analysis}

For the finite-element analysis 2D axisymmetric space dimension model was created in Comsol software. The principle geometry of this model is presented on the following Figure 2.

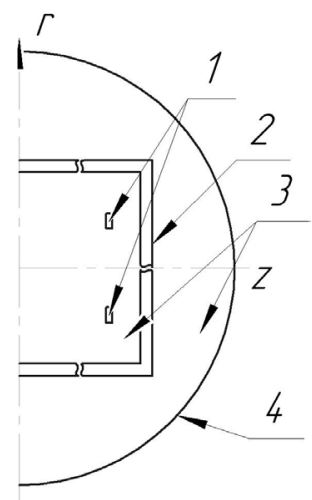

Fig. 2. The principle geometry of the model, where 1 - Helmholtz coils; 2 - magnetic shield; 3 -air domains; 4 - boundaries of the calculation domain.

The magnetic field with stationary computation is used in this model. Parameters of the calculation are presented in the table 1.

Table 1. Parameters of the model.

\begin{tabular}{|l|l|}
\hline Parameters & Parameter value \\
\hline Coils radius $\mathrm{R}, \mathrm{mm}$ & 50 \\
\hline distance $\mathrm{h}, \mathrm{mm}$ & 25 \\
\hline Wire section, $\mathrm{mm}^{2}$ & Square cross section, 0.04 \\
\hline Number of turns & 10 \\
\hline Winding current, $\mathrm{mA}$ & 1 \\
\hline
\end{tabular}

Primarily the value of magnetic flux density in the axial direction without shield and external magnetic field was calculated. According to the simulation results until the value of current in the wind of the coil is equal to $1 \mathrm{~mA}$, the magnetic flux density in the system center is $1795 \times 10^{-7} \mathrm{~T}$ (Figure 3 ).

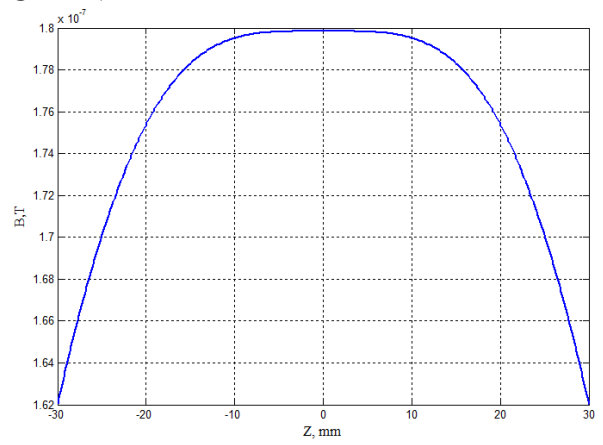

Fig. 3. The magnetic flux density in the axial direction without shield and external magnetic field.

The magnetic field inhomogeneity is calculated by the formula (4). According to the calculation, Helmholtz coils with the parameters from the table 1 provide the needed homogeneity in the interval $[-8.5 ; 8.5] \mathrm{mm}$ (Figure 4). 


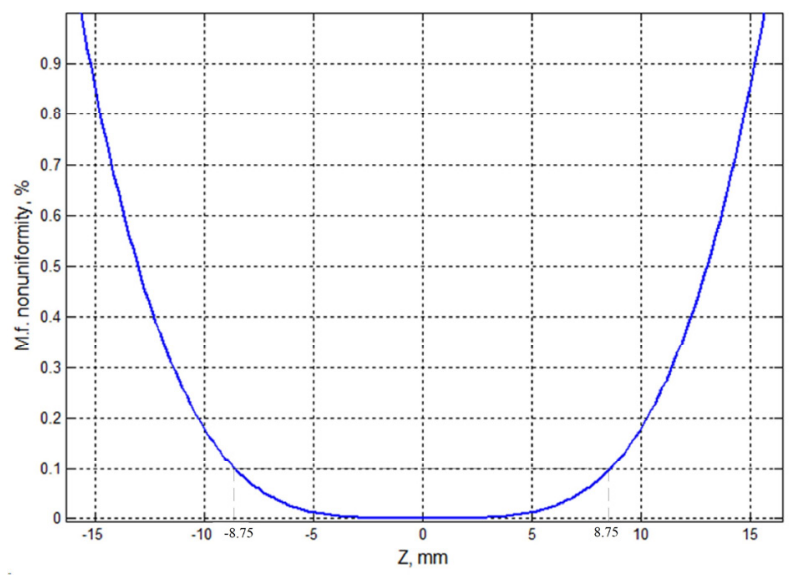

Fig. 4. Magnetic field inhomogeneity for the Helmholtz oils system.

\section{The testing of the shield material and thickness}

\subsection{Iron shield}

For the shielding of the permanent magnetic field the iron shield is used [7]. To define the magnetic shield thickness that allows to provide magnetic shielding of the external magnetic field under the flux density value lesser then $10 \mathrm{nT}$ authors carried out the parametrical analysis to define the dependence of the flux density in the system center with the coil current equals to zero and the iron shield thickness.

Input parameters were:the value of the external magnetic field equals to $50 \mu \mathrm{T}$; the shield is cylindrically shaped with the $\mathrm{r}=100 \mathrm{~mm}$ and the height equals to $200 \mathrm{~mm}$.

According to the calculation the raise of the iron thickness leads to the non-linear reducing of the induction (Figure 5).

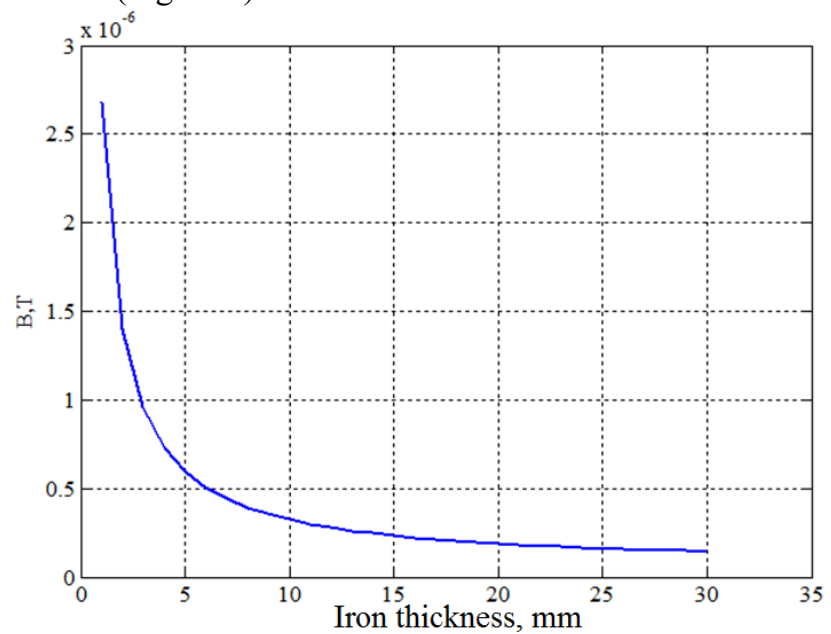

Fig. 5. The value of the magnetic flux density in the shielding system center in the dependence from the shield thickness. 
The Figure 5 shows that if the shield thickness equals to $30 \mathrm{~mm}$ then the external magnetic field falls to the value of $1,5 \mu \mathrm{T}$, that exceeds the value of the field created by the Helmholtz coils with the same parameters.

The resulting magnetic field of the Helmholtz coils system and the external magnetic field is presented on the Figure 6.

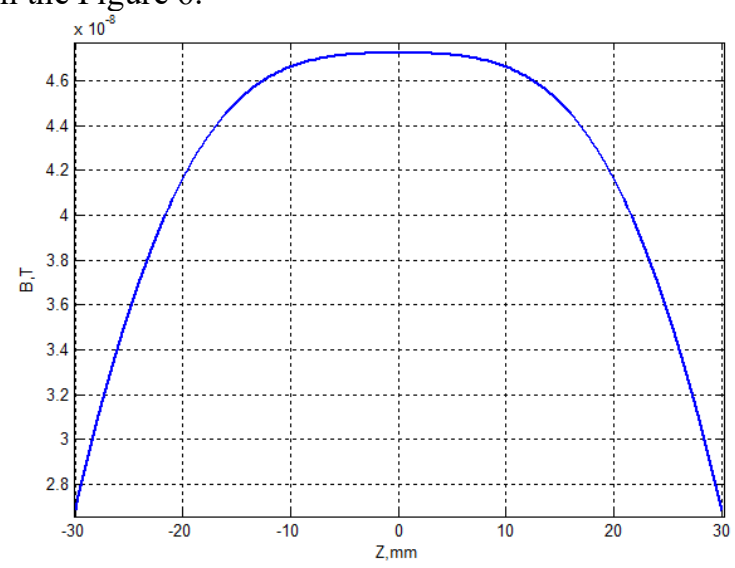

Fig. 6. the magnetic field inhomogeneity with the presence of the external magnetic field and $30 \mathrm{~mm}$ thickness iron shield.

The calculation showed that iron magnetic shield with the thickness $30 \mathrm{~mm}$ allows to provide the needed homogeneity higher than $0.1 \%$ only in the interval $[-2.5 ; 2.5] \mathrm{mm}$. But the iron shield of that thickbess do not allow to provide the sheilding of the external magnetic field under the value of $10 \mu \mathrm{T}$. Thus the using of the iron shield in that case is inappropriate.

\subsection{Finemet shield}

The alloy Finemet is usually used as the magnetic shielding material [8]. The distinctive characteristic of the alloy is its high magnetic permeabilty that defines it as great ferromagnetic shield.

The Figure below shows the dependence of the magnetic flux density in the system center from the Finemet thickness.

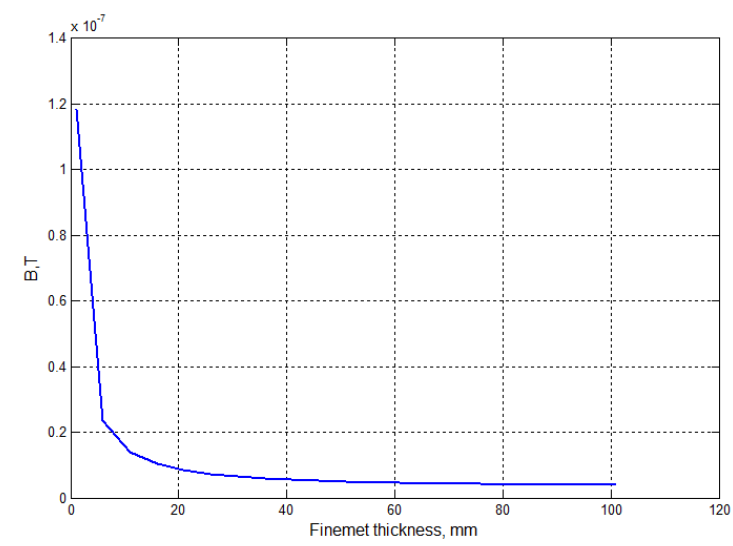

Fig. 7. The dependence of the magnetic flux density in the system center from the Finemet thickness. 
The comparison of the Figures 5 and 7 shows that in the conditions of using the parameters from the table 1 Finemet is more preferable for the magnetic shielding for this model. The parametrical analysis of the shield thickness and the current in the coils windings influence on the magnetic field inhomogeneity. The simulation results are presented on the Figure 8.

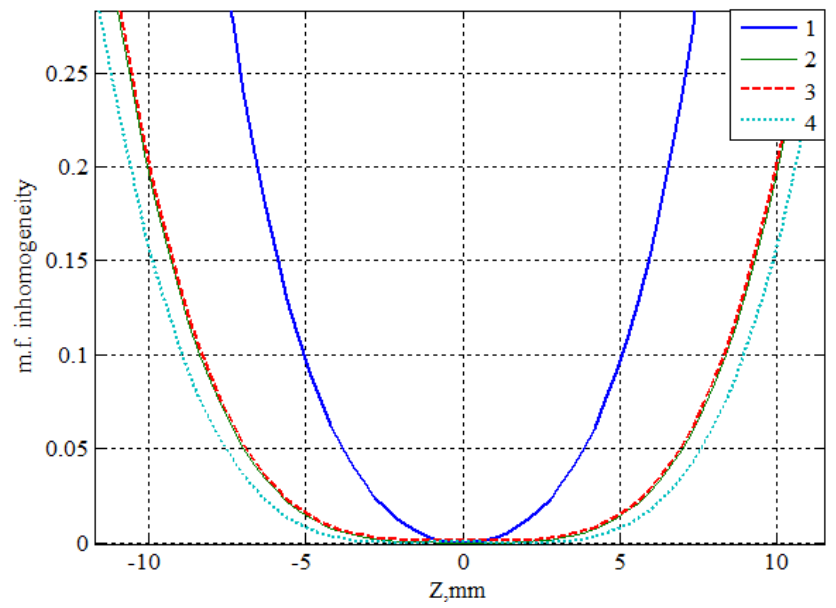

Fig. 8. Helmholtz coil magnetic field inhomogeneity in the situation of availability of external magnetic field and shielding material, where $1-\mathrm{I}=1 \mathrm{~mA}$, shield thickness $=1 \mathrm{~mm} ; 2-5 \mathrm{~mA}, 1 \mathrm{~mm}$; $3-1 \mathrm{~mA}, 5 \mathrm{~mm} ; 4-5 \mathrm{~mA}, 5 \mathrm{~mm}$.

According to the Figure 8, it is apparent that the plot 2 and 3 are coincided and it shows that step-up of the coil in the winding influence the augmentation of the magnetic field homogeneity interval. The system with the current in the coils winding equals to $5 \mathrm{~mA}$ and the shield thickness $-5 \mathrm{~mm}$ has the optimal magnetic field homogeneity. But the magnetic shield with the thickness $5 \mathrm{~mm}$ do not allow to provide magnetic field shielding in the center of the system to the value of $10 \mathrm{nT}$.

According to the Figure 7, to provide the magnetic shielding to the value $10 \mathrm{nT}$ it is necessary to set the $18 \mathrm{~mm}$ thickness of the magnetic shield.

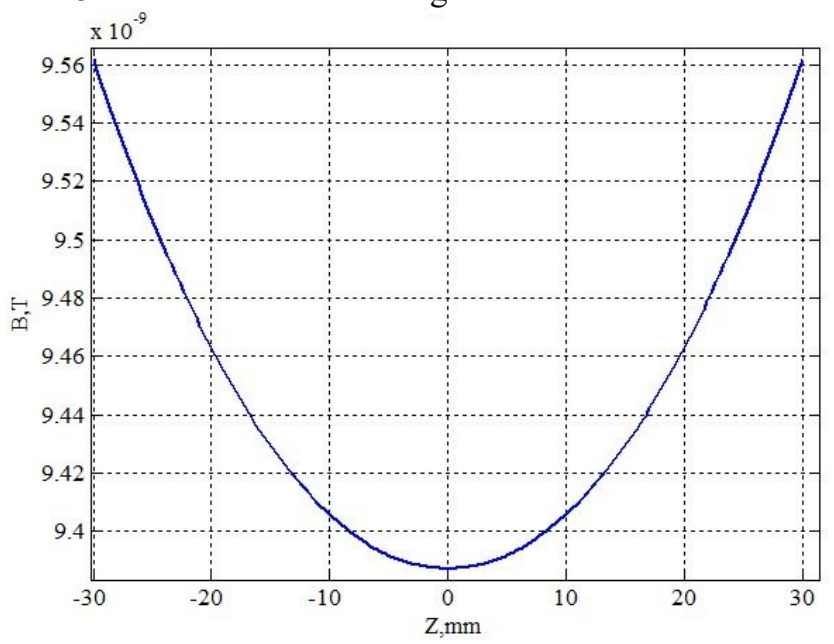

Fig. 9. The magnetic field shielding. 
The Figure 9 shows that the value of the magnetic flux density in the direction of ' $z$ ' in the interval $[-30 ; 30] \mathrm{mm}$ is lesser than the $10 \mathrm{nT}$.

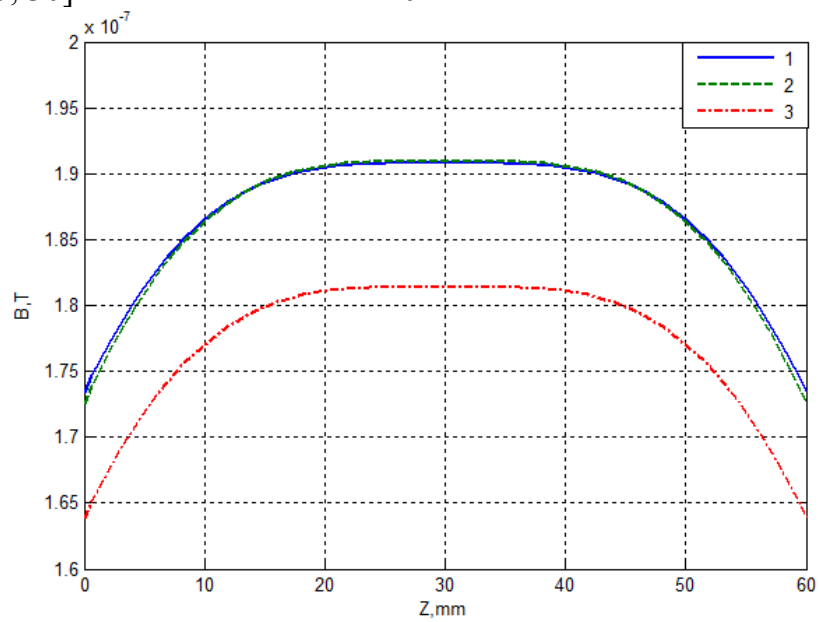

Fig. 10. The magnetic flux density in the axial direction, where $1-$ the external magnetic field is absent, the current in the coils winding $-1 \mathrm{~mA} ; 2$ - the external magnetic field equals to $50 \mu \mathrm{T}$, the current in the coils winding $1.05 \mathrm{~mA} ; 3$ - the external magnetic field equals to $50 \mu \mathrm{T}$, the current in the coils winding $1 \mathrm{~mA}$.

The Figure 10 demonstrates that in the presence of the external magnetic field the magnetic flux density in the axial direction averagely falls on more than $5 \%$. The increasing of the current value in the coil from the $1 \mathrm{~mA}$ to the $1.05 \mathrm{~mA}$ allows to receive the original characteristic.

The Figure 11 below shows the distribution lines of the magnetic flux density in the model.

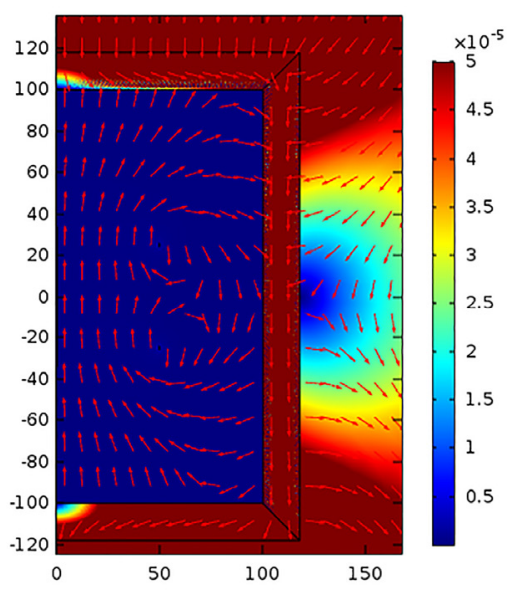

Fig. 11. The distribution of the magnetic field. Shield thickness $18 \mathrm{~mm}$; current in the coils wining $1.05 \mathrm{~mA}$.

The red arrow line shows that magnetic flux density is closed by the magnetic circle in the shield material. It is explain by the high value of Finemet material magnetic permeability. 


\section{Conclusion}

1. The comparison of the Figures 3 and 10(plot \#1) shows that in the absents of external magnetic field the magnetic shield leads to the magnetic flux density increasing in the conditions of the task.

2. The use of the ferromagnetic shield in the case of this simulation allowed to provide external magnetic field shielding to the value lesser than $10 \mathrm{nT}$.

3. The use of the calibrating coils allows to improve the homogeneity on the magnetic field and to create the required magnetic flux density in the shielding system.

\section{Acknowledgments}

The research is funded from Russian Science Foundation (RSF), Grant Number 17-7910083.

\section{References}

[1] P. Ripka, Magnetic science and technologies (Artech house, Boston,2001)

[2] Can, H. \& Topal, U. J. Supercon.\& Nov. Magnet. 28(3),4 (2015)

[3] M.W. Johnson, M.H.S. Amin, S. Gildert, et.al., Nature 473,194 (2011)

[4] V.E. Baranova, P.F. Baranov, Mech.\&Mach., 4 (2014)

[5] Y. Öztürk, B. Aktaş, Rev. Scien. 667,12009 (2016)

[6] V. Ogay, P.Baranov, A. Stepankova, Mat.Scienc. \&Eng. 66, 6 (2014)

[7] A. Keshtkar, A. Maghoul and A. Kalantarnia, In.J. Com.Sc.\&El.Eng. 3 (4), 3(2011)

[8] Hitachi metal [Online] URL: http://www.hitachimetals.com 